\title{
Modified Blumgart Anastomosis in Patients Undergoing Robotic Pancreaticoduodenectomy: The Results of a Preliminary Experience
}

\author{
Marco V. Marino ${ }^{1,2^{*}}$, Adrian Kah Heng Chiow ${ }^{3}$, Gianpaolo Vaccarella ${ }^{1}$, Olexii Potapov ${ }^{4}$, \\ Andrzej Lech Komorowski ${ }^{5}$
}

${ }^{1}$ General and Emergency Surgery Department, Azienda Ospedaliera,

Ospedali Riuniti Villa Sofia-Cervello, Palermo, Italy

${ }^{2}$ General Surgery Department, Istituto Villa Salus, Siracusa, Italy

${ }^{3}$ Hepatopancreatobiliary Unit, Department of Surgery, Changi General Hospital, Singapore

${ }^{4}$ Minimally Invasive Surgery Department, Center for Innovative Medical Technologies of the

National Academy of Sciences of Ukraine, Kiev, Ukraine

${ }^{5}$ General Surgery Department, College of Medicine, University of Rzeszow, Poland

\section{ABSTRACT}

Backgrounds: Several anastomoses have been described for the reconstruction of the pancreatic stump after pancreaticoduodenectomy. No technique showed a superiority over the others in terms of post-operative pancreatic fistula formation (POPF) in open neither during a minimally invasive approach.

Methods: We compared the surgical outcomes of fourteen patients classified as high-risk for POPF who underwent a Blumgart anastomosis (BA) with that of patients who underwent a Cattell-Warren (CW). A 1:1 case-matched analysis was performed according to the variables of the risk score for post-operative pancreatic fistula (POPF) formation.

Results: The overall operative time was similar ( $373 \pm 115$ vs $398 \pm 127$ min, $p=0.567)$ while fashioning of the CW anastomosis required a longer time (38 \pm 21 vs $29 \pm 10$, $p<0.05)$. The overall morbidity rate was comparable among the two groups $(p=0.273)$, while the incidence of clinically relevant-POPF was lower in the BA group (0 vs $3, p<0.05)$. No differences were observed in reoperation rate $(p=0.445)$ and in postoperative length of stay ( 11.5 vs $12.6, p=0.367$ ).

Conclusion: The BA anastomosis is a potential alternative to the $\mathrm{CW}$ technique in a high-risk population for POPF formation. It shows benefits in terms of shorter anastomotic time and lower rate of clinically relevant-POPF.

Key words: robotic pancreatic surgery, robotic pancreaticoduodenectomy, blumgart anastomosis, pancreatico-jejunostomy, pancreatic fistula, surgical outcomes.

\section{INTRODUCTION}

Postoperative pancreatic fistula (POPF) remains the Achilles' heel of pancreaticoduodenectomy, both in open and minimally invasive surgery, ranging from 5 to $20 \%$ of cases, even in high volume pancreatic centers, (1) leading to increased health care costs, prolonged hospital stay (2) and nonnegligible mortality (3).

\author{
*Corresponding author: \\ Marco V. Marino MD, PhD, FEBS, \\ FACS, FICS \\ Salerno place 1, 90127 Palermo, Italy \\ Phone: +39 3389842949 \\ E-mail: marco.vito.marino@gmail.com
}

\author{
Abbreviations: \\ POPF: Postoperative pancreatic fistula, \\ BA: Blumgart anastomosis, \\ CW: Cattell-Warren, \\ PJ: pancreato-jejunostomy, \\ BA: Blumgart anastomosis, \\ ISGPF: International Study Group \\ for Pancreatic Fistula, \\ CT: computed tomography, \\ SD: standard deviation.
}

Received: 05.09.2021

Accepted: 07.11.2021 
Significant efforts have been attempted to identify potential risk factors for POPF after pancreaticoduodenectomy (4) and various modifications of the pancreatic anastomoses have been proposed in the effort to prevent POPF (5-6).

However, there is no universally accepted method and there is no international consensus on how to reconstruct the pancreatic stump after pancreaticoduodenectomy with to minimize the incidence of POPF (7).

The pancreato-jejunostomy (PJ) is the most frequently performed anastomosis after pancreaticoduodenectomy worldwide (8), and currently there are two main variants: the anastomosis of the pancreatic duct to the jejunal mucosa (duct-to-mucosa) with covering parenchymal sutures (Cattell-Warren) (CW) or the invagination of the pancreatic stump into the jejunum (9). A recent meta-analysis showed similar rates of POPF including clinically relevant POPF (CRPOPF) with similar rates of post-operative morbidity and mortality (10). However, heterogeneity of POPF definition, consideration of known risk factors for POPF and technical differences in the performance of these anastomoses continue to make conclusions difficult.

The Blumgart technique (11), and its modified method (12) were proposed as an alternative to the classical duct-to-mucosa pancreatojejunostomy. They combine both a duct-to-mucosa anastomosis and a fullthickness pancreatico-jejunal anastomosis by $\mathrm{U}$-sutures (in effect a mattress stitch), thus allowing the coverage of the pancreatic cut surface with jejunal serosa.

The Blumgart anastomosis (BA) is gaining popularity amongst specialized pancreatic surgeons as it seems to be associated with lower clinically relevant-POPF rate when compared to the previously described pancreatico-jejunostomy (13) or pancreaticogastrostomy (14). Its superiority however was recently be questioned (15).

The comparative studies of the different anastomosis techniques have been generally studied mostly in open comparative series, with limited reports in minimally invasive approaches. The technical challenge of Whipples specifically with duct to mucosa PJ anastomosis in laparoscopy is often considered a major hurdle to adoption.

In this controversial scenario, the use of robotics may be advantageous due to its micro-suturing capabilities, tremor filtration and the advanced degrees of freedom of the instruments, which can improve the precision and the ergonomic during the execution of fine movements such as the fashioning of a challenging duct to mucosa pancreatico-jejunostomy (16).

Despite the potential benefits, the robotic approach for the Blumgart anastomosis (BA) is unfortunately scarcely described. We analyzed the surgical outcomes of our initial experience in robotic BA anastomosis with the aim to assess its safety and feasibility in a group of patients at high risk of POPF. To the authors understanding, this is one of the first reported comparative study between the $\mathrm{CW}$ technique and the BA technique for duct to mucosa PJ reconstruction in robotic Whipples surgery.

\section{PATIENTS AND METHODS}

A retrospective analysis of our prospectively maintained database included 14 consecutive patients who underwent BA during robotic PD was performed from January 2018 to June 2019 at our Tertiary Care Center. The group was case-matched in a 1:1 fashion with patients extracted from our database who underwent robotic $\mathrm{CW}$ reconstruction. The matching was performed according to the following variables: demographic characteristics, soft pancreatic texture, diameter duct size $<3 \mathrm{~mm}$, histopathology and estimated blood loss which are included in externally validated fistula risk score (17).

The rate of clinically relevant (grade B-C) POPF was investigated as primary endpoint, secondary outcomes were: perioperative morbidity and mortality rate, reoperation and readmission rates, postoperative hospital stay.

The Inclusion criteria was the following: patients undergoing elective pancreatoduodenectomy (PD) or pylorus preserving pancreato-duodenectomy (PPPD) via robotic approach for both benign and malignant conditions.

The Exclusion criteria from the study were as follows: patients undergoing extended pancreatoduodenectomy; left, central or total pancreatectomy; concomitant multi-visceral or vascular resection; borderline resectable or locally advanced tumors, previous pancreatic resection, surgery for known chronic pancreatitis, presence of intraperitoneal or extraperitoneal metastases, tumor size $>6 \mathrm{~cm}$, ASA score higher than III, the inability to tolerate pneumoperitoneum, patient refusal for robotic approach.

The work has been conducted in accordance with declaration of Helsinki guidelines. Informed consent was obtained from all participants and the study was approved by the institution's institutional review board.

All procedures were performed by the same surgical team, with good experience in $\mathrm{CW}$ reconstruction during open PD $(n=60)$ and initial in robotic PD $(n=50)$ before commencement of the study timeline. The 
experience in BA reconstruction was limited to open PD $(n=30)$.

\section{Definitions}

The revised International Study Group for Pancreatic Fistula (ISGPF) definition was used to classify the pancreatic fistula (2). Delayed gastric emptying and post-pancreatectomy hemorrhage were classified using the ISGPS definition (18-19).

The pancreatic texture was judged as soft or hard according to the intraoperative assessment and visual clues during the parenchymal transection. The diameter duct size was assessed preoperatively at the level of pancreatic parenchymal transection through magnetic resonance cholangio-pancreatography (MRCP).

Operative time was defined as the time from skin incision to wound dressing. Intraoperative blood loss was quantified by measuring the amount of fluid obtained from the suction device. Postoperative complications were graded according to the ClavienDindo classification system (20) and for patients with multiple complications, the highest grade was considered.

Biliary fistula was defined as drain output with bilirubin concentration of at least three times the serum bilirubin concentration on or after postoperative day three or as the need for radiologic or operative interventions. Mortality was defined as perioperative death within the first 30 days after surgery. Intraabdominal abscess or fluid collection was detected by postoperative computed tomography (CT) scans.

The use of any incision made to perform the pancreatic anastomosis was considered as conversion to open in the robotic group, and patients converted are considered as robotic as intention to treat.

\section{Surgical Technique}

The technique for a robotic-assisted pylorus preserving pancreaticoduodenectomy has been described elsewhere (21). Briefly, the gastrocolic ligament was incised widely and a wide Kocher maneuver was performed. The gastroepiploic vessels were clipped and divided thus exposing the duodenum which is transected with a laparoscopic stapler (blue load). The hepatic hilum dissection was then started, dividing the right gastric artery and finally isolating and clipping the gastroduodenal artery. Cholecystectomy was performed and the common bile duct was encircled and transected above the cystic duct junction. The first jejunal loop was identified and the jejunum transected with a $45 \mathrm{~mm}$ laparoscopic stapler with white reload (Echelon Flex ${ }^{\mathrm{TM}}$, Ethicon, Somerville, NJ, USA). The pancreas was transected with a robot-mounted Harmonic $A C E^{\circledR}$ scalpel, and monopolar curved scissors were used to identify and divide the main pancreatic duct. No somatostatin analogs were intraoperatively administered. An intraoperative frozen section histological examination of the incised margin was performed in all cases. The uncinate process was resected with harmonics scalpel to the right of the superior mesenteric artery and pancreatico-duodenectomy completed. The pancreatic remnant was mobilized at least $2 \mathrm{~cm}$ from the resection margin of the pancreas in preparation for anastomosis. The jejunal limb was brought in the supra-colic compartment by a retro-colic route to the right of middle colic vessels, fashioning an end-to-side anastomoses in a single loop.

\section{Modified Blumgart}

Three to four interrupted U-shaped of 2-0 nonabsorbable suture (Sofsilk ${ }^{\mathrm{TM}}$, Covidien, Dublin, Ireland) or 4-0 monofilament synthetic non-absorbable polypropylene suture (Prolene ${ }^{\mathrm{TM}}$; Ethicon, Somerville, NJ, USA) was placed, transversing the pancreatic parenchyma in full-thickness from its anterior to posterior surface, at least $1 \mathrm{~cm}$ from the section margin of the pancreas without crossing the pancreatic duct, followed by a seromuscular bite with horizontal mattress close to the mesenteric edge of the jejunum. The suture reverted transverse again the whole pancreas from the posterior to the anterior surface at a distance of at least $5 \mathrm{~mm}$ from the initial entry point of the suture completing the U-suture. Each suture is tied before placing the next one and the needles are kept on place at a distance of 5-8 $\mathrm{mm}$.

An inner layer consisting of the duct-to-mucosa anastomosis is performed using a single arm interrupted 5-0 PDS sutures (PDS II $^{\mathrm{TM}}$ Ethicon, Sommerville, NJ, USA).

A trans-anastomotic stenting was placed routinely. An internal 5 French (for duct size smaller than $3 \mathrm{~mm}$ ) or 7 French silastic catheter stent (in case of duct size smaller than $4 \mathrm{~mm}$ ) were inserted into the pancreatic duct to assure its patency.

The needles used for the posterior layer are passed on the anterior seromuscular wall of the jejunum completing the anterior horizontal mattress sutures which are tied on the ventral surface of the jejunum. 


\section{Cattell-Warren}

A posterior row of interrupted monofilament sutures (Prolene 4-0) was placed between the seromuscular layer of the jejunum and the posterior pancreatic capsule. The duct to mucosa anastomosis is performed with a PJ stent similar to the BA anastomosis. An anterior layer of interrupted sutures completes the fashioning of the $\mathrm{CW}$ anastomosis.

An end-to-side hepaticojejunostomy is performed with 5-0 PDS sutures and antecolic end-to-side gastrojejunostomy completed the reconstructive phase.

A routine prophylactic intraperitoneal closed suction drain is placed posteriorly to the PJ and exteriorized through the lateral abdominal wall.

\section{Perioperative Care}

The Antibiotic prophylaxis was started at the time of anesthesia induction and it was continued until postoperative day 3 unless infection was suspected. No prophylactic octreotide was used. Proton pump inhibitors were routinely administered during the hospital stay. Somatostatin analogues (Octreotide) administration was started at $100 \mu \mathrm{g}$ three times a day subcutaneously once a postoperative pancreatic fistula was diagnosed. The nasogastric tube was removed within the first 48 hours after surgery and oral intake progressively increased until soft diet as tolerated.

The amylase levels of serum and its content in the abdominal drains was monitored and measured on postoperative day 3 and 5 . The abdominal drains were removed in the absence of pancreatic fistula on postoperative day 3 (the drain amylase level lower than three times the upper serum amylase activity and daily output was $<150 \mathrm{ml}$ ).

Post-operative consultations at outpatient clinic were scheduled at 3, 6 and 12 months after surgery. A CT-scan is routinely performed 1 month post-operatively.

\section{Statistical analysis}

Continuous variables are expressed as mean values \pm standard deviation (SD). Categorical data are presented as numbers and percentage. The statistical software SPSS version 19.0 (SPSS Inc, Chicago, IL, USA) was used for the statistical analysis.

\section{RESULTS}

A total of twenty-eight patients were included in the present study. The clinical and demographic characteristics of the two groups were comparable (table 1). The most common indication for surgery was pancreatic head adenocarcinoma (17 patients). The post-operative pancreatic fistula risk score was comparable ( $7.6 \pm 1.8$ vs $7.5 \pm 1.6)$.

Despite the overall operative time was similar ( $373 \pm 115$ vs $398 \pm 127 \mathrm{~min}, \mathrm{p}=0.567$ ) the fashioning of the $\mathrm{CW}$ anastomosis required a longer time ( $38 \pm 21$ vs $29 \pm 10, p<0.05$ ) (table 2).

The overall morbidity rate was comparable among the two groups $(p=0.273)$, while the incidence of CR-POPF was lower in the BA group ( 0 vs $3, p<0.05$ ). No differences were observed in reoperation rate $(p=0.445)$ or in postoperative length of stay (11.5 vs 12.6, $\mathrm{p}=0.367)$. There was no inpatient or 30 -day mortality. The oncologic outcomes were comparable in terms of $\mathrm{RO}$ resection rate and number of lymph node harvested.

Table 1 - Demographic and pre-operative characteristics of two case-matched population who underwent $B A(n=14)$ or $C W(n=14)$ reconstruction during robotic pancreatico-duodenectomy

\begin{tabular}{|c|c|c|}
\hline & $B A(n=14)$ & CW $(n=14)$ \\
\hline $\begin{array}{l}\text { Gender, } \mathrm{n} \\
\quad \text { - Male } \\
\text { - Female }\end{array}$ & $\begin{array}{l}8(57.1 \%) \\
6(42.9 \%)\end{array}$ & $\begin{array}{l}7(50 \%) \\
7(50 \%)\end{array}$ \\
\hline Age, year, mean \pm SD & $63.2 \pm 11$ & $64 \pm 13$ \\
\hline $\mathrm{BMI}, \mathrm{kg} / \mathrm{m}^{2}$, mean $\pm \mathrm{SD}$ & $25.2 \pm 3.5$ & $24.9 \pm 3.2$ \\
\hline $\begin{array}{l}\text { ASA score, n } \\
-1 \\
-I I \\
-I I I\end{array}$ & $\begin{array}{l}3(21.4 \%) \\
7(50 \%) \\
4(28.6 \%)\end{array}$ & $\begin{array}{l}3(21.4 \%) \\
6(42.9 \%) \\
5(35.7 \%)\end{array}$ \\
\hline $\begin{array}{l}\text { Comorbidities, n } \\
\text { - Diabetes } \\
\text { - Cardiovascular disease } \\
\text { - Hypertension }\end{array}$ & $\begin{array}{c}1(7.1 \%) \\
2(14.3 \%) \\
1(7.1 \%)\end{array}$ & $\begin{array}{l}2(14.3 \%) \\
1(7.1 \%) \\
1(7.1 \%)\end{array}$ \\
\hline Previous abdominal surgery, $\mathrm{n}$ & $2(14.3 \%)$ & $2(14.3 \%)$ \\
\hline $\begin{array}{l}\text { Preoperative biliary drainage, } \mathrm{n} \\
\text { - Percutaneous } \\
\text { - Endoscopic retrograde }\end{array}$ & $\begin{array}{c}3(21.4 \%) \\
1(7.1 \%)\end{array}$ & $\begin{array}{c}2(14.3 \%) \\
1(7.1 \%)\end{array}$ \\
\hline $\begin{array}{l}\text { Pathology, n } \\
\text { - Pancreatic Head Adenocarcinoma } \\
\text { - Ampullary Adenocarcinoma } \\
\text { - IPMN Main-duct } \\
\text { - Mucinous Cystic Neoplasm } \\
\text { - Neuroendocrine Carcinoma } \\
\text { - Duodenal Carcinoma }\end{array}$ & $\begin{array}{c}8(57.1 \%) \\
2(14.3 \%) \\
2(14.3 \%) \\
1(7.1 \%) \\
1(7.1 \%) \\
-\end{array}$ & $\begin{array}{c}9(64.3 \%) \\
1(7.1 \%) \\
1(7.1 \%) \\
1(7.1 \%) \\
1(7.1 \%) \\
1(7.1 \%)\end{array}$ \\
\hline Tumor size, $\mathrm{cm}$, mean $( \pm \mathrm{SD})$ & $2.71 \pm 1.7$ & $2.75 \pm 2.1$ \\
\hline Neoadjuvant CHT-RT, n & $2(14.3 \%)$ & $3(21.4 \%)$ \\
\hline Callery Risk score, mean $( \pm S D)$ & $7.6 \pm 1.8$ & $7.5 \pm 1.6$ \\
\hline Main pancreatic duct-size, mm, mean $( \pm \mathrm{SD})$ & $2.55 \pm 0.3$ & $2.59 \pm 0.7$ \\
\hline $\begin{array}{l}\text { Pancreatic texture, } \mathrm{n} \\
\text { - Soft } \\
\text { - Hard }\end{array}$ & $\begin{array}{c}12(85.7 \%) \\
2(14.3 \%)\end{array}$ & $\begin{array}{c}12(85.7 \%) \\
2(14.3 \%)\end{array}$ \\
\hline
\end{tabular}


Table 2 - Post-operative outcomes of the two case-matched populations who underwent BA $(n=14)$ or $C W(n=14)$ during a robotic pancreatico-duodenectomy

\begin{tabular}{|c|c|c|c|}
\hline Variable & $B A(n=14)$ & PJ $(n=14)$ & $P$ value \\
\hline Operative time, min, mean \pm SD & $373 \pm 115$ & $398 \pm 127$ & 0.567 \\
\hline Time of Anastomosis, min, mean \pm SD & $29 \pm 10$ & $38 \pm 21$ & $<0.05$ \\
\hline Blood loss, $\mathrm{mL}$, mean $\pm \mathrm{SD}$ & $335 \pm 90$ & $320 \pm 80$ & 0.648 \\
\hline Intraoperative Blood transfusion, $\mathrm{n}$ & l & $1(7.1 \%)$ & \\
\hline $\begin{array}{l}\text { Morbidity rate, } \mathrm{n}(\%) \\
\quad \text { - Minor }(\text { Clavien }<3) \\
\text { - Major (Clavien } \geq 3 \text { ) }\end{array}$ & $\begin{array}{c}4(28.6 \%) \\
-3 \\
-1\end{array}$ & $\begin{array}{l}7(50 \%) \\
-2 \\
-5\end{array}$ & 0.273 \\
\hline CR-POPF & 1 & $3(21.4 \%)$ & $<0.05$ \\
\hline Biochemical Leak, n & $1(7.1 \%)$ & $1(7.1 \%)$ & 1 \\
\hline Intrabdominal collection & $1(7.1 \%)$ & $1(7.1 \%)$ & 1 \\
\hline Delayed Gastric Emptying, grade B & $1(7.1 \%)$ & $1(7.1 \%)$ & 1 \\
\hline Surgical site Infection & $1(7.1 \%)$ & l & \\
\hline $\mathrm{PPH}$, grade $\mathrm{C}$ & l & $1(7.1 \%)$ & \\
\hline Length of stay, days, mean \pm SD & $11.5 \pm 7$ & $12.6 \pm 9$ & 0.367 \\
\hline Reoperation, $n$ & l & $2(14.3 \%)$ & 0.445 \\
\hline Readmission, n & $1(7.1 \%)$ & $1(7.1 \%)$ & 1 \\
\hline 30-days Mortality rate, $n$ & 1 & I & N.A \\
\hline R0 resection rate, $n$ & $13 / 14(92.9 \%)$ & $13 / 14(92.9 \%)$ & 1 \\
\hline Lymph Nodes harvested, $n$, mean \pm SD & $27.5 \pm 6$ & $28.4 \pm 7$ & 0.699 \\
\hline
\end{tabular}

\section{DISCUSSION}

Since its first description (11) the Blumgart technique has been progressively adopted by a growing number of pancreatic surgeons both in open (22) and in laparoscopic pancreatic surgery (23).

The potential advantages include the firmly anchoring between the pancreatic gland and the jejunum which act as a coverage on the pancreatic stump, the possibility to perform an accurate and careful tension-free placement of the duct-to-mucosal sutures before securing the posterior and anterior seromuscular of the jejunum (24). This technique eliminated the tangential sutures and minimized the tension and shear force at the pancreatic stump particularly during the tightening of the knots which can lacerate pancreatic parenchyma especially in soft pancreases (14).

The modified Blumgart anastomosis further reduced the number of the trans-pancreatic jejunal seromuscular U-shaped sutures (one to three) which were considered a potential factor which can compromise the blood flow in the pancreatic stump and it introduces the novelty of tying the sutures at the ventral wall of the jejunum (12).

Since 2018 we adopted the BA to our current practice for pancreatic reconstruction after robotic $P D$ in patients considered high-risk for POPF (diameter duct size $<3 \mathrm{~mm}$ and soft pancreatic texture) due to the increasing number of evidences which supported the potential advantages of this reconstruction.

Despite the potential advantages over the standard Cattell-Warren anastomosis, several authors reported contradictory results. Kleepies et al showed that the BA seems to minimize overall complications ( $15 \%$ vs $31 \%$, $\mathrm{p}=0.015$ ) and pancreatic leak rate ( $4 \%$ vs $13 \%, p=0.03$ ) (21). However, a recent study by Hirano et al showed that these techniques failed to demonstrate their superiority in terms of POPF reduction (BA $10.3 \%$ vs PJ $6.8 \%, p=0.367)$ and in terms of severe complications (17.8\% vs $11.7 \%, p=0.212$ ) when compared with pancreatico-jejunostomy interrupted suture (15).

In a retrospective analysis, Oda et al. concluded the modified BA anastomosis was associated with a lower CR-POPF (20.5\% vs $37.2 \%, p=0.033$ ) and lower length of stay (16 vs 23 days, $p<0.001$ ) when compared to the Kakita method (25) Conversely, the same two techniques did not show any significance difference in CR-POPF when analyzing the outcomes of 282 patients with soft pancreas (26). There is a significant heterogeneity in the applications of both the BA and their comparative technique, which make comparisons between studies difficult and interpretation of systematic reviews and meta-analysis of $\mathrm{PJ}$ anastomosis outcomes need to interpreted in this context.

Furthermore, the robotic pancreaticoduodenectomy, being an emerging technique, is still confined to highselected pancreatic centers; therefore, the robotic $B A$ is 
scarcely reported (27-28). In this study we aimed to assess the safety and efficacy of the BA in a selected patient population with soft pancreatic glands and small diameter duct size, in which the BA may have a unique benefit since the same suture is used to buttress the anterior and posterior pancreatic surface.

According to our experience, the lack of tactile feedback was not a pitfall when we dealt with soft pancreases as the visual clues offered by the enhanced visualization of the robotic platform allowed us to assess a correct tissue apposition. In particular we observed a clear advantage in fashioning the duct-tomucosa anastomosis due to the fact that the posterior capsule of the pancreas was not anchored to the seromuscular layer of the jejunal wall.

The manipulation of several stitches which were left uncut, was in our hands, one of the potential drawbacks of the technique which require deep experience in tissue handling and an expert bedside assistant.

The overall time for fashioning the BA anastomosis was $27 \mathrm{~min}$ which is considerably faster when compared to that needed during our experience in $\mathrm{CW}$ anastomosis despite this being the author's early experience with this anastomosis robotically (29). The reduction in operative time is also confirmed by the experience of other authors who reported a reduction in operation time (386.4 VS $473.1 \mathrm{~min}, \mathrm{p}<0.001$ ) of the BA when compared to Cattell-Warren (13). Furthermore, there was a significant difference in rate of CR-POPF although this did not translate to a significant difference in post-operative length of stay and overall morbidity.

In our patient population, the surgical morbidity was acceptable (21.4\%) and only one patient experienced severe complication. These data, despite compromised by the small sample size, seems to confirm the safety of this anastomosis which was reported to reduce severe complications ( 0 versus $35.1 \%, p<0.001$ ) with respect to $\mathrm{CW}$ anastomosis and invagination $\mathrm{PJ}$ and reoperation rate $(2.7 \%$ vs $16.2 \%, p=0.056)$ with respect to $C W$ anastomosis (30).

It is likely that a well conducted multicenter prospective study is required before assessing the superiority of one technique over the other, and the first prospective multicenter randomized controlled trial (PANasta trial) whose aim is to compare the BA versus Cattell-Warren anastomosis and to assess the impact on pancreatic fistula formation is currently underway may eventually answer the question (31). In parallel, it is conceivable that a parallel study should be attempted in a series of patients undergoing minimally invasive PD or PPPD as the technical challenges in performance of these anastomoses can be different compared to the open technique.

Our study has some limitations being retrospective and single-center in nature, the small sample size and the performance bias due to the learning curve of the surgeons involved, but at the same time, it is one of the few studies who investigated the surgical outcomes of the robotic BA in a selected patient population.

\section{CONCLUSION}

In conclusion, The BA anastomosis is a potential alternative to the $\mathrm{CW}$ technique in high POPF risk pancreaticojejunostomy with a benefit of shorter anastomotic time and lower CR-POPF. Further prospective studies in other minimally invasive pancreaticoduodenectomy patients comparing these techniques are needed to confirm our early results.

\section{Disclosure statement}

All authors have no conflict of interest to disclose. Dr. Marino is Assistant Medical Director of CAVA Robotics LLC.

\section{Funding}

This research did not receive any specific grant from funding agencies in the public, commercial, or notfor-profit sectors.

\section{REFERENCES}

1. McMillan MT, Soi S, Asbun HJ, Ball CG, Bassi C, Beane JD et al. Risk-adjusted Outcomes of Clinically Relevant Pancreatic Fistula Following Pancreatoduodenectomy: A Model for Performance Evaluation. Ann Surg. 2016;264(2):344-352.

2. Bassi C, Marchegiani G, Dervenis C, Sarr M, Hilal MA, Adham M et al. The 2016 update of the International Study Group (ISGPS) definition and grading of postoperative pancreatic fistula: 11 Years After. Surgery. 2017;161(3):584-591.

3. Asbun HJ, Stauffer JA. Laparoscopic vs open pancreaticoduodenectomy: overall outcomes and severity of complications using the Accordion Severity Grading System. J Am Coll Surg. 2012;215(6):810-819

4. Fu SJ, Shen SL, Li SQ, Hu WJ, Hua YP, Kuang M et al. Risk factors and outcomes of postoperative pancreatic fistula after pancreaticoduodenectomy: an audit of 532 consecutive cases. BMC Surg. 2015;15:34

5. Peng SY, Wang JW, Lau WY, Cai XJ, Mou YP, Liu YB et al. Conventional versus binding pancreaticojejunostomy after pancreaticoduodenectomy: a prospective randomized trial. Ann Surg. 2007;245(5):692-698.

6. Kakita A, Takahashi T, Yoshida M, Furuta K. A simpler and more reliable technique of pancreatojejunal anastomosis. Surg Today. 1996;26(7):532-535

7. Cheng $\mathrm{Y}$, Briarava M, Lai M, Wang XM, Tu B, Cheng NS et al. Pancreaticojejunostomy versus pancreaticogastrostomy reconstruction for the prevention of postoperative pancreatic fistula following pancreaticoduodenectomy. Cochrane Database Syst Rev. 
2017;9(9):CD012257.

8. Klaiber U, Probst P, Knebel P, Contin P, Diener MK, Buchler MW et al. Meta-analysis of complication rates for single-loop versus dual-loop (Roux-en-Y) with isolated pancreaticojejunostomy reconstruction after pancreaticoduodenectomy. Br J Surg. 2015;102(4): 331-340.

9. Senda $Y$, Shimizu $Y$, Natsume $S$, Ito $S$, Komori K, Abe $T$ et al. Randomized clinical trial of duct-to-mucosa versus invagination pancreaticojejunostomy after pancreatoduodenectomy. Br J Surg. 2018;105(1):48-57.

10. Zhang $\mathrm{S}$, Lan Z, Zhang J, Chen $\mathrm{Y}, \mathrm{Xu} Q$, Jiang $Q$ et al. Ductto-mucosa versus invagination pancreaticojejunostomy after pancreaticoduodenectomy: a meta-analysis. Oncotarget. 2017; 8(28):46449-46460.

11. Grobmyer SR, Kooby D, Blumgart LH, Hochwald SN. Novel pancreaticojejunostomy with a low rate of anastomotic failurerelated complications. J Am Coll Surg. 2010;210(1):54-59.

12. Fujii T, Sugimoto $H$, Yamada $S$, Kanda M, Suenaga M, Takami $H$ et al. Modified Blumgart anastomosis for pancreaticojejunostomy: technical improvement in matched historical control study. Gastrointest Surg. 2014;18(6):1108-1115.

13. Lee YN, Kim WY. Comparison of Blumgart versus conventional ductto-mucosa anastomosis for pancreaticojejunostomy after pancreaticoduodenectomy. Ann Hepatobiliary Pancreat Surg. 2018;22(3): 253-260.

14. Wang SE, Chen SC, Shyr BU, Shyr YM. Comparison of Modified Blumgart pancreaticojejunostomy and pancreaticogastrostomy after pancreaticoduodenectomy. HPB (Oxford). 2016;18(3):229-235.

15. Hirono S, Kawai M, Okada KI, Miyazawa M, Kitahata Y, Hayami S et al. Modified Blumgart Mattress Suture Versus Conventional Interrupted Suture in Pancreaticojejunostomy During Pancreaticoduodenectomy: Randomized Controlled Trial. Ann Surg. 2019; 269(2):243-251.

16. Marino MV, Shabat G, Potapov O, Gulotta G, Komorowski AL. Robotic pancreatic surgery: old concerns, new perspectives. Acta Chir Belg. 2019;119(1):16-23.

17. Callery MP, Pratt WB, Kent TS, Chaikof EL, Vollmer CM Jr. A prospectively validated clinical risk score accurately predicts pancreatic fistula after pancreatoduodenectomy. J Am Coll Surg. 2013;216(1):1-14.

18. Wente MN, Bassi C, Dervenis C, Fingerhut A, Gouma DJ, Izbicki JR et al. Delayed gastric emptying (DGE) after pancreatic surgery: a suggested definition by the International Study Group of Pancreatic Surgery (ISGPS). Surgery. 2007;142(5):761-8

19. Wente MN, Veit JA, Bassi C, Dervenis C, Fingerhut A, Gouma DJ et al. Postpancreatectomy hemorrhage (PPH): an International Study Group of Pancreatic Surgery (ISGPS) definition. Surgery. 2007;
142(1):20-5

20. Clavien PA, Barkun J, de Oliveira ML, Vauthey JN, Dindo D, Schulick $\mathrm{RD}$ et al. The Clavien-Dindo classification of surgical complications: five-year experience. Ann Surg. 2009;250(2):187-96

21. Marino MV, Podda M, Gomez Ruiz M, Fernandez CC, Guarrasi D, Gomez Fleitas M. Robotic-assisted versus open pancreaticoduodenectomy: the results of a case-matched comparison. J Robot Surg. 2020;14(3):493-502.

22. Kleespies A, Rentsch M, Seeliger H, Albertsmeier M, Jauch KW, Bruns CJ. Blumgart anastomosis for pancreaticojejunostomy minimizes severe complications after pancreatic head resection. $\mathrm{Br}$ J Surg. 2009;96(7):741-750.

23. Poves I, Morató 0, Burdío F, Grande L. Laparoscopic-adapted Blumgart pancreaticojejunostomy in laparoscopic pancreaticoduodenectomy. Surg Endosc. 2017;31(7):2837-2845.

24. Mishra PK, Saluja SS, Gupta M, Rajalingam R, Pattnaik P. Blumgart's technique of pancreaticojejunostomy: an appraisal. Dig Surg. 2011;28(4):281-287.

25. Oda T, Hashimoto S, Miyamoto R, Shimomura 0, Fukunaga K, Kohno $\mathrm{K}$ et al. The Tight Adaptation at Pancreatic Anastomosis Without Parenchymal Laceration: An Institutional Experience in Introducing and Modifying the New Procedure. World J Surg. 2015;39(8):2014-2022.

26. Kawakatsu S, Inoue Y, Mise Y, Ishizawa T, Ito H, Takahashi $Y$ et al. Comparison of pancreatojejunostomy techniques in patients with a soft pancreas: Kakita anastomosis and Blumgart anastomosis. BMC Surg. 2018;18(1):88. Published 2018 Oct 24.

27. Beane JD, Zenati M, Hamad A, Hogg ME, Zeh HJ 3rd, Zureikat AH. Robotic pancreatoduodenectomy with vascular resection: Outcomes and learning curve. Surgery. 2019;166(1):8-14.

28. Liu R, Wakabayashi G, Palanivelu C, Tsung A, Yang K, Goh BK et al. International consensus statement on robotic pancreatic surgery. Hepatobiliary Surg Nutr. 2019;8(4):345-360.

29. Marino MV, Podda M, Pisanu A, di Saverio S, Fleitas MG. Roboticassisted Pancreaticoduodenectomy: Technique Description and Performance Evaluation After 60 Cases. Surg Laparosc Endosc Percutan Tech. 2020;30(2):156-163.

30. Casadei R, Ricci C, Ingaldi C, Alberici L, De Raffele E, Minni F. Comparison of Blumgart Anastomosis with Duct-to-Mucosa Anastomosis and Invagination Pancreaticojejunostomy After Pancreaticoduodenectomy: A Single-Center Propensity Score Matching Analysis. J Gastrointest Surg. 2021;25(2):411-420. Epub 2020 Jan 29.

31. Halloran CM, Platt K, Gerard A, Polydoros F, O'Reilly DA, Gomez D et al. PANasta Trial; Cattell Warren versus Blumgart techniques of pancreatico-jejunostomy following pancreato-duodenectomy: Study protocol for a randomized controlled trial. Trials. 2016;17:30. 